\title{
Simulated vs video-assisted learning on clinical competency and retentivity; a preliminary study
}

\author{
Azizan, A.B.B., Subramaniam, T., Yan, L.S., Wen, T.Y., Sivanendran, D.A.P.S., Kirihettige, \\ D.R.G. \\ International Medical University, Malaysia
}

\section{Background:}

Educating medical students exclusively in the hospital setting poses a challenge in view of the COVID19 pandemic. We sought to compare the effectiveness of simulation and video-assisted learning of clinical skills, and retentivity.

\section{Methods:}

This is a randomized single blinded comparative study that explored the outcome of two modalities to teach about airway adjuncts and their application. The simulation group had hands-on learning on a part-task trainer guided by a lecturer while the video-assisted group watched a demonstration on a part task by the same lecturer. The learning content was the same. Students' performance on knowledge and practical skills was compared using one best answer questions (OBA) and direct observation of procedural skills (DOPS) respectively. We observed their performance over time; immediate, 2 weeks and 8 weeks post exposure to determine retentivity. VARK questionnaire was used to identify learning styles and the relationship with both teaching modalities.

\section{Results:}

Mann-Whitney $U$ test showed a significant difference in the third DOPS assessment $(U=17.50, p=$ 0.011 ). No difference was noted in OBA scores. Friedman test was performed to assess retentivity, followed by a Wilcoxon signed-rank test which showed a significant decrease in the first to the third OBA scores in the video group $(Z=-2.038, p=0.042)$, unlike the simulation group which showed no change. Both groups showed no change in performance in the DOPS assessments.

\section{Conclusions:}

Our findings indicate that video-assisted learning may be as equally effective as simulated learning in acquiring knowledge on simple airway devices. There appeared to be no relationship between learning styles and performance in both groups. Both groups showed retention in their clinical skills. However, the video-assisted group showed decay of knowledge over time while the simulation group did not. Further research with bigger sample size and more complex devices would be needed to support our findings.

Keywords: Clinical skills, Simulation, Video-Assisted, Knowledge 